\title{
Analysis and Recognition Method of Internet Image Public Opinion Based on Partial Differential Equation
}

\author{
Yang Zhang $\mathbb{D}$ \\ Business School, University of Shanghai for Science and Technology, Shanghai 200093, China \\ Correspondence should be addressed to Yang Zhang; 171910094@st.usst.edu.cn
}

Received 16 August 2021; Revised 26 August 2021; Accepted 28 August 2021; Published 15 September 2021

Academic Editor: Miaochao Chen

Copyright (C) 2021 Yang Zhang. This is an open access article distributed under the Creative Commons Attribution License, which permits unrestricted use, distribution, and reproduction in any medium, provided the original work is properly cited.

\begin{abstract}
This article comprehensively and systematically expounds the development trends and basic theory of partial differential methods, analyzes the characteristics of sampling multiscale transformation in detail, and deeply studies the network image denoising and network image restoration methods that perform partial differential diffusion in the pixel domain and the transform domain. An adaptive diffusion method of partial differential equations is proposed. Among them, the key parameters can be adaptively changed according to the curvature and gradient of the local geometric information of the network image, and the diffusion direction and intensity of the diffusion can be controlled. First, using the principle of variation, we derive the Euler equation corresponding to the diffusion method of partial differential equations and analyze its diffusion ability using the local orthogonal coordinate system of the network image. Based on the theoretical analysis of public opinion, this article applies opinion mining technology to the online public opinion early warning system to achieve the purpose of grasping the opinions of netizens in time and guiding the trend of public opinion. Opinion mining is the use of natural language processing technology to automatically extract the emotional tendencies and evaluation objects contained in the subjective text. In the edge area of the network image, the diffusion along the edge direction should have a large diffusion coefficient, and the diffusion along the vertical edge direction should have a small diffusion coefficient; in the flat area of the network image, it diffuses to the surrounding with equal intensity, and the diffusion intensity value is relatively high. Secondly, based on the analysis of the adaptive partial differential equation diffusion method, using the half-point difference format, a numerical method for network image recognition is designed. Both theoretical analysis and experimental results show that the network image recognition model based on adaptive partial differential equation diffusion is more effective than the model based on partial differential equation recognition; at the same time, experiments show that the network image recognition model based on adaptive partial differential equation diffusion is more effective than the network image recognition model based on ordinary diffusion. The network image recognition model based on constant partial differential equation diffusion is more effective in improving the quality of network image recognition.
\end{abstract}

\section{Introduction}

The partial differential method has been widely used in the fields of network image processing, signal processing, and network image compression, but its huge amount of calculation restricts the further promotion of the application of the partial differential method [1]. Combining the partial differential method with the multiscale analysis method and using the respective advantages of the two methods to form a new network image processing method can greatly reduce the calculation time spent by the partial differential method, which is of great theoretical and practical value [2]. At present, the partial differential method has been applied to the fields of edge extraction, feature extraction, pattern recognition, network image segmentation, machine vision, etc., showing a good application prospect [3]. However, the existing partial differential method is sensitive to noise, which limits its application in the field of network image noise reduction and network image recognition to a certain extent [4]. In the process of network image acquisition and transmission, it is often interfered by noise, and the purpose of noise reduction is to remove the noise in the signal while 
maintaining the main characteristics of the original signal as much as possible and improve the quality of the network image [5]. In the process of network image storage and transmission, part of the network image may be damaged or part of the compression coefficient may be lost, resulting in a serious decline in network image quality. Only the method of network image noise reduction cannot solve the problem of network image imaging quality, so partial recognition is carried out by using partial differential network image recognition method [6].

In network image processing and computer vision, the systematic use of partial differential equation (PDE) methods is an emerging field developed in the past decade [7]. It has accumulated rich research results and demonstrated strong vitality. On the one hand, it has benefited from partial differential equations as an important branch of mathematics and has formed a relatively mature theoretical system and numerical methods; on the other hand, it has benefited from the experience accumulated in the traditional network image technology [8]. The network image recognition method based on partial differentiation provides new implementation methods and methods for network image noise reduction, network image recognition, and blind restoration of network images. It is currently one of the most active frontier research topics in the field of network image processing [9]. The main purpose of digital network image recognition is to make it impossible for the observer to perceive that the network image has been modified or get a better visual effect. It is mainly used in network image coding, network image transmission, network image compression, and identification of pictures, hidden objects, archaeology, etc. [10]. Its main working principle is to use the known pixel information around the to-be-recognized area of the digital network image to fill the to-be-recognized area of the digital network image [11]. Network image recognition is different from network image filtering. The noise network image contains both effective and noise signals, while the area to be recognized in the network image does not contain any meaningful points. The area to be recognized can only be estimated from the edge information of the area to be recognized. The pixel value is limited by the technical conditions of network image processing, although the network image quality cannot be accurately identified, the network image recognition method based on partial differentiation can greatly improve the quality of the network image [12].

This paper proposes an adaptive recognition method of network image public opinion based on partial differential equations. First, the network image is nonlinearly sampled and transformed to obtain transformation coefficients in different scales and directions, and then, the coefficients of decomposition scales and regional energy are used to represent the network. This paper mainly studies the key technologies of opinion mining based on online public opinion and applies opinion mining to the public opinion early warning system, which makes up for the shortcomings of the existing public opinion early warning system. The paper introduces the existing opinion mining technology for product reviews from different levels and proposes a fine-grained Internet opinion mining technology based on dynamic knowledge base. At the beginning of the evaluation, it is necessary to classify and evaluate the emotional impact of these three evaluation criteria on the information recipient. When the evaluation subject is evaluating, if the focus is on the outcome of the event, then the goal is the most important; if the evaluation subject pays more attention to the behavior of the object in the event, then the code of conduct should be more important. This technology is different from the traditional opinion mining process. It uses clustering method to extract the topic of opinion sentences and dynamically joins the knowledge base. With the improvement of the knowledge base, the factors of manual intervention will become smaller and smaller, and it has better adaptability. Then, according to the characteristics of the transform coefficients, the direction factor of the nondownsampling transform is introduced to adaptively determine the noise reduction threshold. Finally, the transform coefficients are inversely transformed to achieve network image noise reduction.

\section{Related Works}

Generally speaking, the network image restoration process is a complex digital network image processing process, including network image noise reduction, network image enhancement, network image motion blur estimation, network image recognition, and network image nonlinear filtering [13]. Depending on the imaging mechanism and the cause of network image degradation, different processing methods can be used. At present, the theory of network image processing methods mainly includes three categories: methods based on stochastic modeling, methods based on wavelet and multiscale analysis, and partial differential equations, among which wavelet and multiscale analysis methods are used. It is most active in network image denoising; in network image recognition and blind restoration, the research based on partial differential methods is the most active [14]. In recent years, the above methods have been combined with each other, and many successful examples of network image processing have been obtained.

Hou et al. [15] proposed that the heat conduction equation is not the only equation that can form a scale space. In fact, the evolution equations that satisfy the maximum value principle can define the scale space, and the maximum value principle is the mathematical expression of the causality criterion, thus proposing a scale space. The anisotropic diffusion proposed by Shan et al. [16] is the most influential in this field. They propose to replace the Gaussian diffusion with a selective diffusion that can maintain the edge to preserve the edge information. The equation uses the conductivity coefficient to modulate the space. The amount of diffusion of each point makes the diffusion mainly occur in each area of the network image. As the diffusion progresses, the interior of the lowcontrast area becomes smoother and the high-contrast area (such as the edge) is maintained. The network image gradient diffuses in the orthogonal direction and can also produce diffusion in the network image gradient direction. However, the selection of the conductivity coefficient in the equation lacks a theoretical basis for PM diffusion, and PM diffusion is essentially ill-conditioned, that is, the equation is solved for uniqueness and stability of numerical calculation cannot be 
guaranteed. Jost and his research team [17] proposed geometrically restricted partial differential equations, the most famous of which is curvature flow. Curvature flow is a "pure" anisotropic diffusion model, which makes the gray value diffusion of network images only occur. In the orthogonal direction of the network image gradient, smooth denoising is performed along the contour while maintaining the precise position and definition of the network image contour. In addition, studying on shock waves and total variational denoising has shown the advantages of partial differential equations in processing network images. The success of these methods lies in treating network images as sliced smooth functions (surfaces) connected by jumping edges. This is related to the piecewise smooth solution of a certain partial differential equation, which highlights the importance of partial differential equations in network image processing.

Evans and Kelley [18] revised the KNN (K Nearest Neighbor) algorithm in the topic tracking technology based on the classification algorithm and proposed a topic tracking algorithm based on topic updates, pointing out that the KNN algorithm has a priori report sparsity aspect. The problem of classification defects was brought by the construction of a tree structure, the topic category is the root, and the training sample is the leaf. During training, the number of leaf nodes is constantly adjusted to make the training sample evenly distributed. In this way, when the $\mathrm{KNN}$ algorithm is used for classification, it will be able to meet the requirement of uniform distribution of its training samples. The paper by Chaturvedi et al. [19] pointed out that the key problem of topic tracking is to establish a model that reflects the characteristics of topic structure and topic deformation. When analyzing different topic models, it provides a sudden incremental learning method and an update of the time series event chain. Finally, after comprehensively analyzing the performance of various topic models, a core capture attenuation evaluation standard based on feature overlap ratio is proposed. The article points out that the vector space model is a basic model for describing static topics based on a packet of words, while the dynamic topic model introduces an adaptive learning mechanism on the basis of the static topic model. The incremental learning algorithm realizes the topic model from the static topic to dynamic model. The paper by Lewandowsky et al. [20] improved topic tracking technology from the aspect of feature weight calculation method and proposed a calculation method based on improved weight. The article weights the weight value of the feature item according to the different position of the feature item in the document. The feature item of the first sentence of the article uses the second highest weighted weight, and the other parts of the article feature items use the general feature weight calculation method [21].

\section{Network Image Public Opinion Analysis and Recognition Model Construction Based on Partial Differential Equations}

3.1. Spatial Distribution of the Solution Set of Partial Differential Equations. Partial differential equations are derived from basic natural laws, applied mathematics, mathematical physics, and engineering technology. Many important physical problems can be described by partial differential equations and corresponding initial conditions, boundary conditions, or initial-boundary mixed conditions. Figure 1 shows the spatial distribution level of the partial differential equation solution set.

The filter corresponding to this equation has a sharpening effect, which can make the blurred network image, especially the edge part, become more obvious, but this may also create some false edges.

$$
\begin{gathered}
X=\left\{x \in L^{2}, \nabla x \in L^{2}\right\}, \\
\int f(x)^{2} d x+|x-x(0)| d x=0 .
\end{gathered}
$$

Using partial differential equations to deal with network image problems, it is difficult to obtain an analytical solution, and the numerical approximation (or approximate) solution is generally sought. There are many commonly used methods for numerical solution of partial differential equations, such as finite difference method, finite element method, and boundary element method, among which the finite difference method is the most widely used.

$$
\begin{gathered}
\frac{\partial x}{\partial t}=t \times(x-x(0))+\nabla \cdot(\nabla x(t)-x(0)), \\
\arg \min C(x)=t * f(x, x(0))+f(x) .
\end{gathered}
$$

This equation derives the AMSS (Affine Morphological Scale Space) operator, which satisfies multiple invariances such as translation invariance, gray translation invariance, affine invariance, and mathematical morphology invariance. It is the best type of filter used in network image processing to meet various requirements for smoothness.

$$
\begin{gathered}
u(x, t)=\left\{\begin{array}{l}
(t-1) \times \frac{\partial x}{\partial t}, x, t \in D, \\
0, x, t \notin D,
\end{array}\right. \\
\frac{\partial f(x)}{\partial x}+\frac{\partial x(t)}{\partial t}=0 .
\end{gathered}
$$

The situation of two-dimensional functions is much more complicated than one-dimensional. In practice, twodimensional differential operators generally use cell template convolution to perform approximate calculations. The gradient of a two-dimensional function is defined as follows:

$$
\begin{gathered}
\operatorname{div}\left[s(|\nabla u(x)|) \times \frac{\nabla u(x)}{|\nabla u(x)|}\right]-t(u(x)-u(0))=0, \\
\frac{\partial u(x)}{\partial x}-\nabla u(x)^{2}|x \times(\nabla u(x)-u(0))|=0 .
\end{gathered}
$$

According to the size of the template and the different values of the elements (coefficients), many different operators have been proposed. The operator uses a similar 


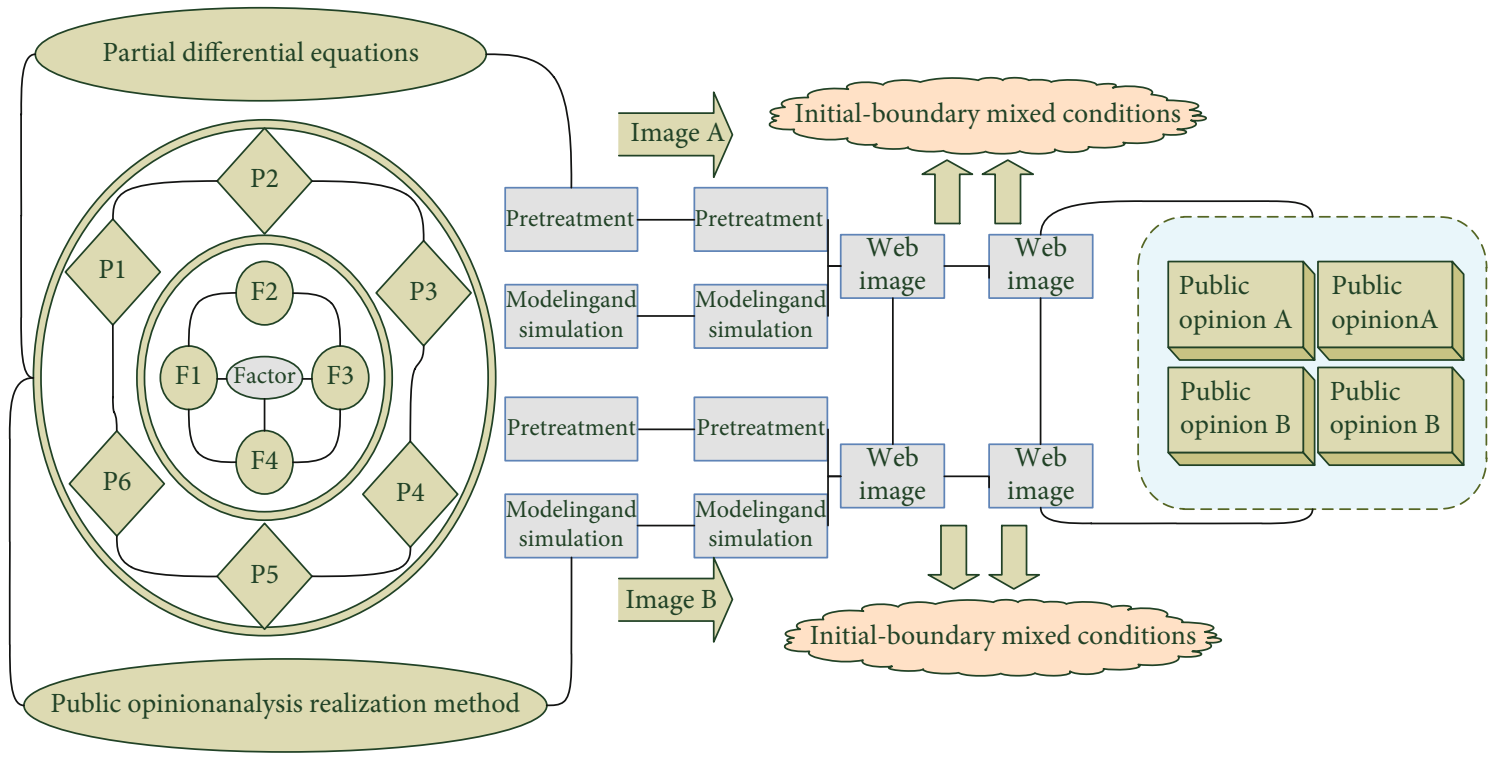

Figure 1: The spatial distribution level of the solution set of partial differential equations.

convolution method to move the template on the network image and calculate the gradient value of the corresponding center pixel at each position.

3.2. Network Image Recognition Algorithm. Online public opinion warning involves multiple disciplines, the most important of which is natural language processing technology. NLP (natural language processing) is a technology that uses natural language to communicate with computers. Because the key to processing natural language is to allow computers to "understand" natural language, natural language processing is also called NLU (natural language processing).

$$
\begin{gathered}
\left\{\begin{array}{l}
\frac{\partial u(x)}{\partial x}=u_{x}, \\
\frac{\partial^{2} u(x)}{\partial x^{2}}=u_{x x}, \\
\frac{\partial^{2} u(x, y)}{\partial x \partial y}=u_{x y},
\end{array}\right. \\
\frac{\partial A(x, y)}{\partial x}=\frac{\partial A(x, y)}{\partial u(x)} u_{x x}+\frac{\partial A(x, y)}{\partial u(y)} u_{y x}, \\
\frac{1}{|\nabla u(x, y)|} \times(u(x), u(y))=\frac{\nabla u(x, y)}{|\nabla u(x, y)|} .
\end{gathered}
$$

From the perspective of natural language understanding, processing text requires three steps: (1) understanding each word that appears, (2) constructing a structure that expresses the meaning of a sentence from the meaning of the word, and (3) expressing the structure of speech from the semantic structure of a sentence. In these three processes, we need to focus on how to effectively use grammar, semantics, pragmatics, and various related knowledge issues. The purpose of region segmentation of the network image is to divide the network image into several disconnected regions, so that the gray level (or other attributes) of the network image in each region is basically the same, but the gray level of the network image between regions is basically the same. Figure 2 shows a schematic diagram of network image recognition.

We describe the network image segmentation problem in mathematical language as follows: for a given network image $u(x, y)$, we find an optimized piecewise smooth approximation function $g(x, y)$ of $f(x, y)$ and the region. Decomposition makes $g(x, y)$ smooth in each region, it is discontinuous or has a large gradient at the boundary of the region.

$$
\frac{\left(u_{x x} u_{y}^{2}-2 u_{x y} u_{x} u_{y}+u_{y y} u_{x}^{2}\right)}{|\nabla u(x, y)|^{2}}=1 .
$$

The diffusion equation needs to meet the following characteristics: (1) causality: when the scale changes from small to large, no new detailed features are produced. The heat conduction equation is causal, that is, as the scale $t$ increases, the smoothing effect of the network image is enhanced, and the details in the original network image gradually disappear. (2) Positioning characteristics: at different scales, the spatial position changes of network image features are positioning characteristics. The thermal conduction equation has poor positioning characteristics, that is, the retained network image features on a large scale will widen and drift in space.

$$
\sigma^{2}=\frac{1}{\Omega} \times \iint(u(x, y)-u(0))^{2} d x d y
$$

To solve this problem, the main features of the network image are first detected on a large scale, and then, the scale is gradually reduced, and the features are tracked on a small scale. In this way, the main features can be more accurately positioned. However, this method is very complicated and unstable. Different smoothing methods are adopted according 


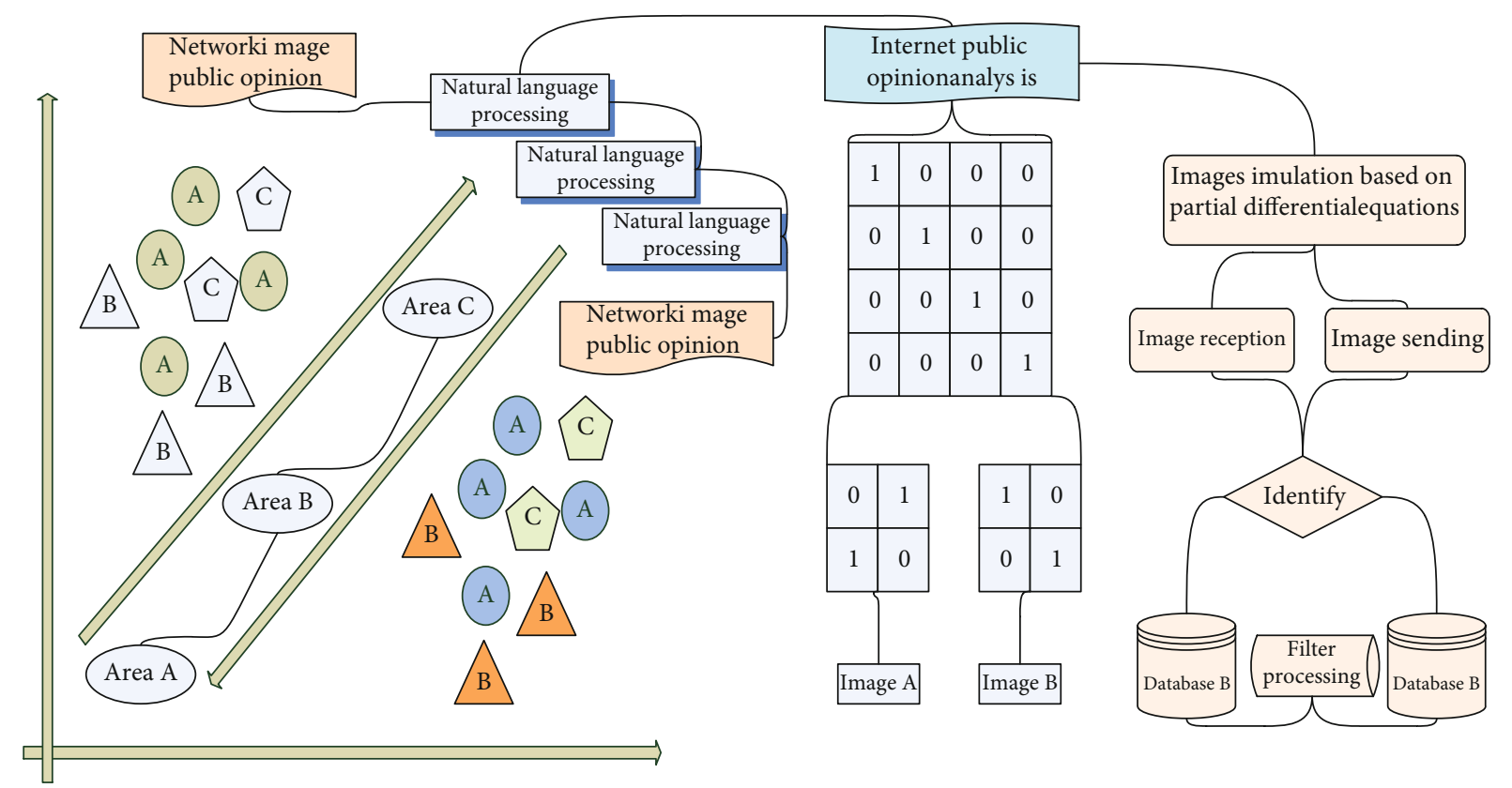

FIGURE 2: Schematic diagram of network image recognition.

to different network image content. The sampling element has two parameters: the receptive field and the threshold. The receptive field is used to determine the number of connected units, and the threshold is used to control the degree of response to the characteristic subpattern. Therefore, the partial differential equation network is generally composed of layers and layers alternately, and finally, full connection is used to complete specific tasks. This special network structure has three structural characteristics: local connection, weight sharing, and subsampling in space or time. Intuitively speaking, this technology can weaken the intensity of smoothing at the edge position and increase it in the nonedge area; at the same time, it smooths in the direction parallel to the edge and does not smooth in the direction perpendicular to the edge, so that it is better while smoothing the noise.

$$
H(x, y)=\left[\begin{array}{ccc}
u_{x x} & 0 & 0 \\
0 & u_{x y} & 0 \\
0 & 0 & u_{y y}
\end{array}\right]
$$

This type of filter can maintain the edge to a large extent while denoising. Therefore, the anisotropic nonlinear diffusion filter actually changes adaptively according to the result of the last network image smoothing. This reflects that the anisotropic nonlinear diffusion filter has feedback to the last smoothing result, and this feedback can be used to guide a new smoothing process. Therefore, this type of filter has the advantages that the classic template filter algorithm does not have.

$$
\left[\begin{array}{ll}
1 & 0 \\
0 & 1
\end{array}\right] \times\left[\begin{array}{ll}
u_{x x} & u_{x y} \\
u_{y x} & u_{y y}
\end{array}\right]=(u(x), u(y))^{T} .
$$

In the classic network image edge detection technology based on gradient or zero-crossing edge operator, the sensitivity to noise and the difficulty of extracting closed edges are the main disadvantages of this type of technology. In addition, this type of technology independently determines edge points in different local areas of the image, and it is difficult to effectively use the global information of the edge and the knowledge of the target to be divided. Using deformation surface technology based on partial differential equations, such as active contour algorithm and level set method, can better overcome the shortcomings of unclosed edges and noise sensitivity in classic edge detection algorithms.

3.3. Optimization of Public Opinion Analysis Factors. The representative technology for the new event recognition type is the single-pass clustering (SPC) algorithm. The single-pass clustering algorithm treats each document extracted from the document stream as a separate category to form a topic cluster, and each topic cluster is represented by the average vector of members, that is, the centroid; for each subsequent input document, there are two thresholds for the similarity with each centroid: one is called the clustering threshold, and the other is called the innovation threshold. The clustering threshold is greater than the innovation threshold. The document belongs to this topic; if the similarity between the current input document and a topic cluster is less than the clustering threshold but greater than the innovation threshold, then the document is discarded and not considered; if the current input document is similar to a topic cluster, the similarity is less than the innovation threshold, then the document is judged to be a new topic, and a new topic with it as the center of mass is created. Figure 3 shows the three-dimensional histogram distribution of the similarity of network image public opinion under different thresholds. When calculating document similarity, the improved single- 
pass clustering uses time window technology to correct the similarity between the current topics $a, b$, and $c$ by using the temporal adjacency between news reports belonging to the same topic value.

According to some report samples, we find other reports that belong to a certain topic; the goal of topic tracking is to filter tasks similar to information retrieval, providing a small number of reports known to be on the same topic and requesting to find out the reports in the stream. For all reports on this topic, the method of topic tracking is to extract a feature set from the training report, which can ensure that the training report is different from a larger set of reports; when a new report appears, it will be compared with the topic features; if enough, it means it is about this topic.

Table 1 shows the collection attributes of the topic clusters of the Internet public opinion. The input is a collection of news documents sorted in chronological order. Initially, the document collection is divided into topic clusters composed of each single document; we perform clustering operations on each new cluster and continuously merge the old clusters (low-level clusters) in each new cluster. The concept of dynamic knowledge base is integrated into opinion mining, and based on synonyms, clustering method is used to realize fine-grained opinion topic mining. The number of clusters in it is reduced to a predetermined number, or the similarity between each of the two clusters is lower than a predetermined threshold; so far, the clustering operation in the new cluster is ended; all current clusters are arranged in order and repeated with $m$ is the division of the unit, and a new round of clustering is performed; until the number of new clusters reaches the predetermined number, the hierarchical topic cluster structure is output.

\section{Application and Analysis of Network Image Public Opinion Analysis and Recognition Model Based on Partial Differential Equations}

4.1. Extraction of Partial Differential Equation Parameters. The mean value filter completes the filtering by replacing the average value of the neighboring pixels with the value of the center pixel, which reduces the sharp change of the gray value in the network image. Generally, the performance of noise in network images is the change of gray level, so in simple terms, denoising is to smooth the gray level. However, the sharp changes in gray level also include the edge information of the network image, so the network image after the mean filtering process is likely to cause edge blur. Tensor diffusion can determine the diffusion speed along the edge direction and the vertical edge direction according to the edge direction to achieve denoising along the edge direction, so as to achieve the purpose of edge enhancement, and overcome the shortcomings of nonlinear diffusion equations that do nothing for edge noise. Figure 4 shows the network image filtering processing flow based on partial differential equations.

In this paper, the standard network image with the size of $512 * 512$ pixels and the highest gray level of 256 is proc-

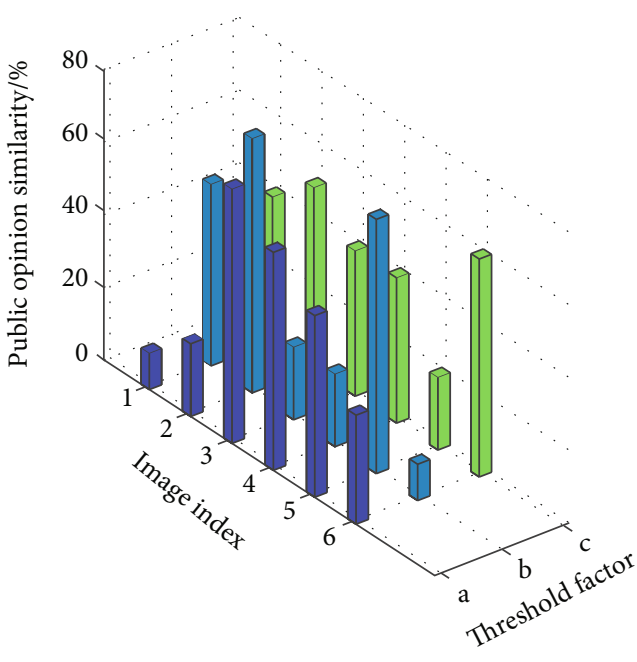

FIgURE 3: Three-dimensional histogram distribution of network image public opinion similarity under different thresholds.

TABle 1: Attributes of online public opinion topic clusters.

\begin{tabular}{lcccc}
\hline $\begin{array}{l}\text { Text } \\
\text { index }\end{array}$ & $\begin{array}{c}\text { Number of } \\
\text { clusters }\end{array}$ & $\begin{array}{c}\text { Diffusion } \\
\text { coefficient }\end{array}$ & $\begin{array}{c}\text { Average } \\
\text { error }\end{array}$ & $\begin{array}{c}\text { Signal-to- } \\
\text { noise ratio }\end{array}$ \\
\hline 1 & 10 & 5 & 5.12 & 54.12 \\
2 & 20 & 10 & 4.53 & 47.01 \\
3 & 30 & 5 & 4.28 & 48.23 \\
4 & 40 & 10 & 4.87 & 51.07 \\
\hline
\end{tabular}

essed by the original P-M diffusion equation. It can be seen that the standard network image is contaminated by noise, the entire network image is full of noise, and the details of the network image are partially damaged. The difference between the Web public opinion corpus and the product review corpus is analyzed, and a new opinion mining idea is proposed according to the characteristics of the Web public opinion corpus. After the diffusion equation is processed, the noise is mostly smoothed, but the effect on the preservation of details is not very good, which affects the judgment of the details. Since the fuzzy threshold is related to the degree of diffusion of $\mathrm{P}-\mathrm{M}$, it is possible to compare and observe the data by adjusting the value.

Table 2 shows the analysis of network image denoising data. The processing results of the above methods using objective data analysis are shown in the text. From the data in the table, it can be seen that the greater the variance of the noise is, the weaker the ability of these methods to deal with network image noise is. The above several network image filtering methods are relatively basic methods in network image denoising. Wavelet coefficients can be obtained by wavelet transformation of the signal, and the signal and noise have corresponding coefficients. For useful signals, due to the uneven spatial distribution, they only exist in certain specific positions and have larger amplitudes, while the amplitudes at other positions are very small, so that all wavelet coefficients have the influence of noise, and the useful information is only on a few wavelet coefficients. Therefore, different coefficients can be distinguished by setting an 


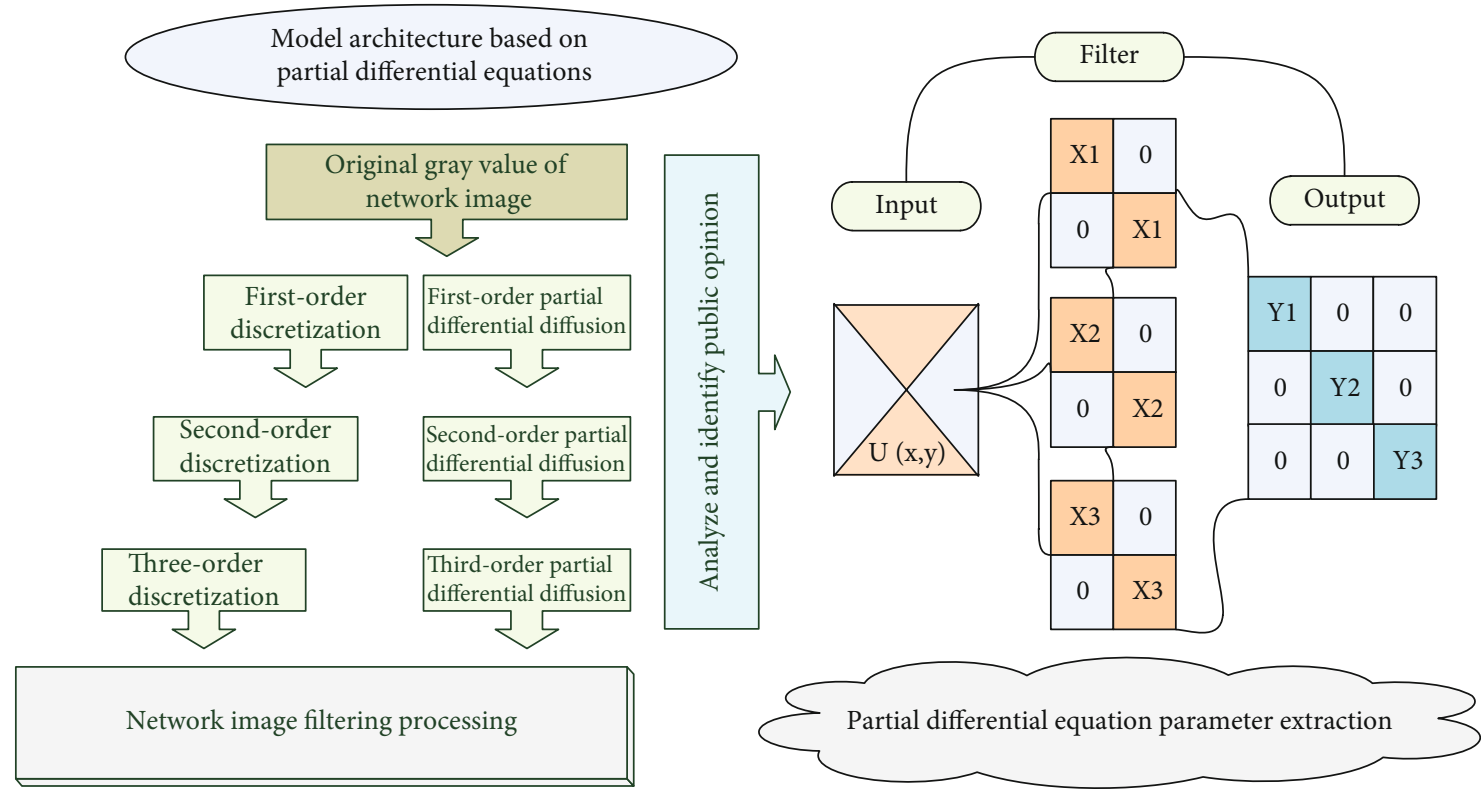

FIGURE 4: Network image filtering processing flow based on partial differential equations.

TABLe 2: Analysis of network image denoising data.

\begin{tabular}{lccc}
\hline $\begin{array}{l}\text { Image } \\
\text { index }\end{array}$ & $\begin{array}{c}\text { Fuzzy } \\
\text { threshold }\end{array}$ & $\begin{array}{c}\text { Noise value/ } \\
\mathrm{dB}\end{array}$ & $\begin{array}{c}\text { Standard } \\
\text { deviation }\end{array}$ \\
\hline 1 & 16.92 & 29.31 & 10.01 \\
2 & 23.01 & 29.43 & 9.97 \\
3 & 21.08 & 30.07 & 9.11 \\
4 & 22.31 & 26.11 & 11.02 \\
5 & 19.82 & 28.31 & 10.82 \\
\hline
\end{tabular}

appropriate threshold value. The noise coefficient with a small amplitude is directly ignored, and the coefficient with a large absolute value is considered as the coefficient of the useful signal.

\subsection{Network Image Public Opinion Recognition Simulation.} The experiments in this article are simulated in Matlab R2016a environment. In order to verify the effectiveness of the hybrid PDE method based on region segmentation in removing noise, we take the network image of the network public opinion and intercept a part of $512 \times 512$ on it. For Gaussian noise, we take the filter power $n=2$ and cut-off frequency $d=1$ and use the method proposed in this chapter to experiment on some public opinion network images after adding noise. In the boundary area, the diffusion method is shown as reverse diffusion protection or even strengthening the boundary; in the inner flat area of the network image, the value of the boundary detection function is basically close to 0 at most points. It is this self-adaptive principle that enables the network image to perform edge detection while diffusing in different ways. The research needs of this article are more inclined to understand the negative comments in emergencies and do not need too much attention for specific negative emotions. Therefore, based on the consideration of fine emotion granularity and closed-loop principle, this article

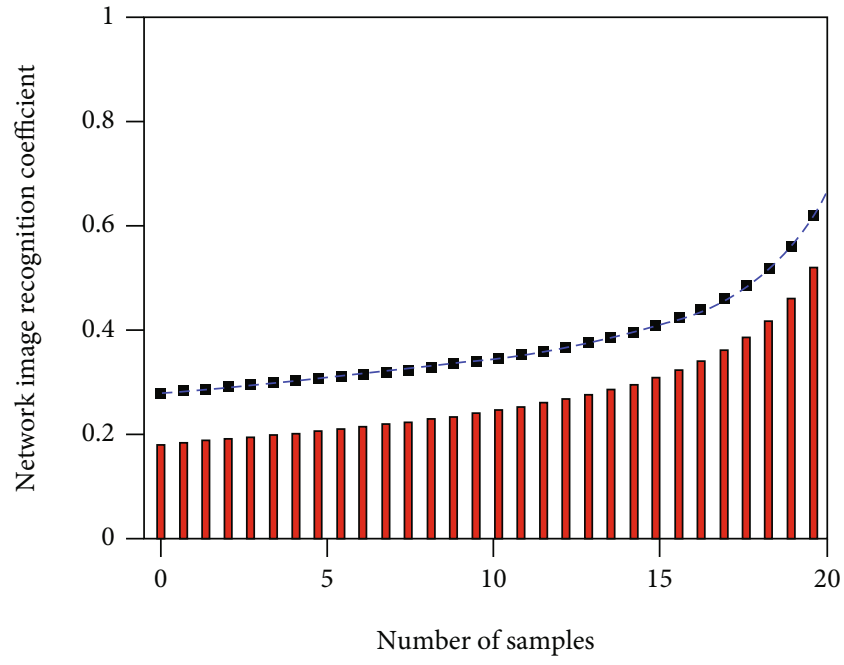

FIGURE 5: Distribution curve of public opinion recognition degree of network images.

innovatively tries to use the deep learning algorithm of positive and negative emotion three classification to recognize the emotion of the case text of network public opinion emergencies. It can well protect the boundary information while removing noise, so that the details of the network image can be preserved and the information of the network image recognition is higher. Figure 5 shows the distribution curve of public opinion recognition of network images.

Internet public opinion sentiment classification based on partial differential equations is actually to use the Weibo text in the emergency case library as a point in the $n$-dimensional feature space, and we use support vector machines to find a hyperplane in this multidimensional feature space to combine different emotions. The types of network public opinion information texts should be separated as much as possible, 


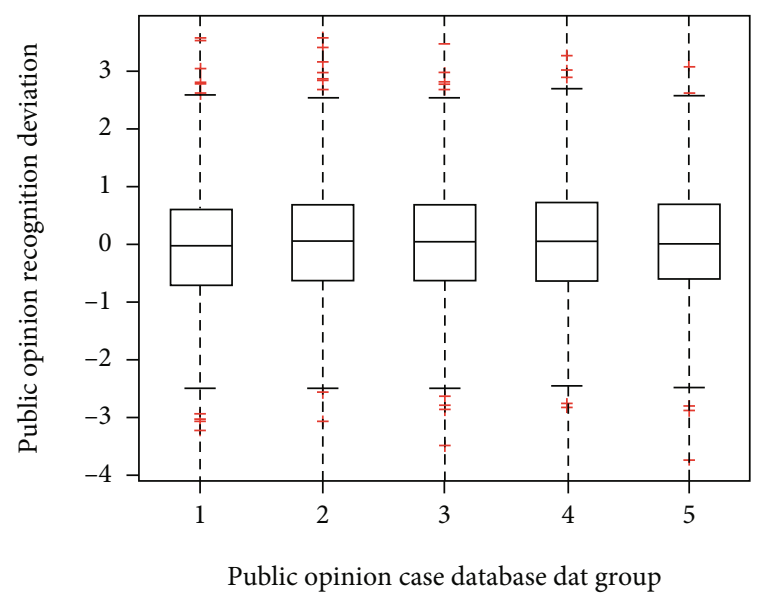

FIgURE 6: Box diagram of network public opinion data identification deviation.

and the space between various types of texts should be as large as possible. After filtering and cleaning the data collected by the crawler, the project team members who understand the emotional rule system are sent to classify and label the Weibo information text of the emergent network public opinion to form a case of the network public opinion emergency incident. Box diagram of network public opinion data identification deviation is shown in Figure 6.

In the input processing of the network, this article quoted the PDE model to train the data set of the case library to generate the training model bin file and then generate the word vector of each word by loading the bin file. In order to compare the effect of word vector generated on sentiment classification, this paper mentions vocabulary index to complete the word embedding operation. We build a vocabulary index table and map each word to between 0 and $n$ ( $n$ is the size of the vocabulary), that is, each word is represented by a specific integer, and each sentence becomes a vector of integers. Numerical experiments show that the improved model has a better denoising effect. While effectively smoothing the network image noise, it also better protects the edge and texture details of the network image.

4.3. Example Application and Analysis. Given a $512 \times 512$ standard network image, the highest gray value of the network image is 256 , and the network image is contaminated by Gaussian noise. It can be seen from the experimental results that the hard threshold method is inferior to the soft threshold method in eliminating noise. This is due to its own discontinuity: although the soft threshold is improved in eliminating noise, the soft threshold is improved. The threshold is not perfect in the preservation of details in the denoising process, which makes the details missing. In order to verify the effectiveness of the method proposed in this chapter for denoising public opinion network images, this chapter uses public opinion network images for verification. Figure 7 shows the evaluation of the public opinion recognition effect of network images for different data points.

The influence of the parameter on the denoising results can be seen; using different smoothing result of the noise network image, data points are set as 15, 20, 25, 35, and 50 , and other parameters remain unchanged. After many debugging and verification, the value of recognition rate is more appropriate from 20 to 50 . The experimental results show that the algorithm can improve the contrast and clarity of public opinion network images and at the same time can better suppress the noise of network images. It can be seen that the image is severely blurred while the noise is removed; using the result of local mean filtering, it can be seen that the edge details of the image are maintained well. It is easy to find that some large noise points are not effectively removed. Although the network image noise is effectively removed, the detailed information of the network image is lost. Figure 8 shows the recognition deviation of the image public opinion feature information matchstick illustration. The new model uses the variance and gradient of the network image as a measure of the local information of the network image, which can better distinguish the different feature information of the network image.

Because different training data, parameters, and models will affect the word vector, it is necessary to determine the appropriate word vector representation dimension according to the actual sentiment of the text information of the emergency case. In this paper, the dimension of word vector representation is set to $100,200,300,400$, and 500, and other hyperparameters and variables are controlled unchanged, and training is performed separately. Figure 9 shows the pie chart of the accuracy of different public opinion training data test sets. It is the accuracy result of the test set after training with different word vector dimensions for three types of emergencies.

Analysis of objective data shows that the threshold method performs better in the data when the noise variance of the two methods is small, but as the noise variance becomes larger, the difference between the soft threshold and the hard threshold function method becomes smaller and smaller. This PDE method can adaptively detect the edge of the network image and divide the network image into smooth areas and boundary areas, so that the diffusion mode of the equation can be controlled adaptively. Due to the adaptability of this diffusion algorithm, it overcomes the traditional equation problem and it can protect the image details very well.

\section{Conclusion}

Aiming at the problem of network image restoration, this paper proposes a new network image blind restoration algorithm based on anisotropy and nonlinear regularization of adaptive partial differential equation diffusion. The algorithm mainly combines the properties of the gradient and curvature of the network image and adopts an anisotropybased spatial adaptive regularization process and establishes a regularization function with nonlinearity and spatial anisotropy, so that it can be adaptive when recovering the target network image gradient smoothing and edge preservation. The cost function is minimized through alternate minimization schemes, and the nonlinear equation is linearized through fixed-point iterative strategies to quickly 


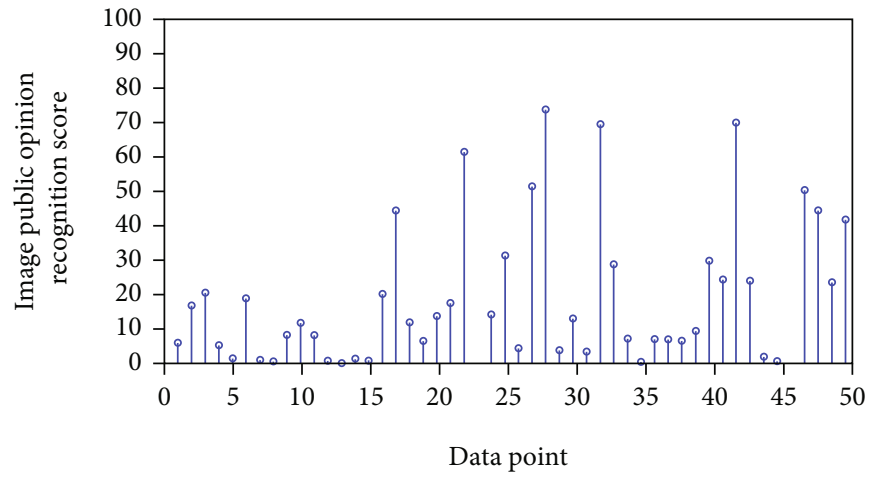

FIGURE 7: Evaluation of the effect of network image public opinion recognition on different data points.

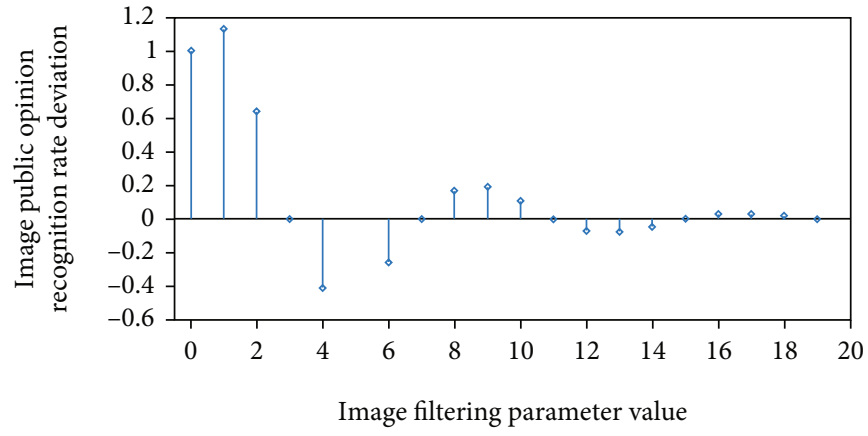

FIGURE 8: Image public opinion feature information recognition deviation matchstick diagram.

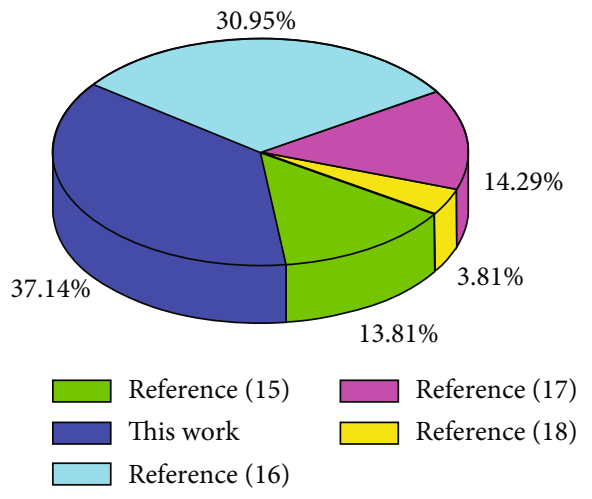

FIGURE 9: Fan chart of accuracy of different public opinion training data test sets.

restore the network image. Based on the characteristics of the online public opinion corpus, this paper proposes a fine-grained online public opinion mining method based on a dynamic knowledge base. This method can not only analyze the overall emotional tendency of Internet users to an online public opinion event, but also effectively mine opinions and comments. In terms of sentiment tendency analysis, this paper constructs a sentiment dictionary with a novel structure, which takes into account the influence of part of speech on the sentiment value of words. At the same time, a voting strategy-based sentiment analysis method is also proposed. This method combines the influence of degree adverbs, negative words, and punctuation marks to more accurately calculate the sentiment orientation value of netizens' opinions. Finally, this article applies the proposed method to the network public opinion early recognition system and obtains good results, which proves the effectiveness of the method proposed in this article. Because some coefficients may be adaptive in the process of network image compression, storage, and transmission, the method proposed in the paper can directly identify coefficients in the transform domain. Both theoretical analysis and experimental results show that the recognition method based on the transform domain is effective, and even when a large number of coefficients are lost, the recognition can be achieved well, and the quality of the network image is obviously improved.

\section{Data Availability}

The data used to support the findings of this study are available from the corresponding author upon request.

\section{Conflicts of Interest}

The authors declare that they have no known competing financial interests or personal relationships that could have appeared to influence the work reported in this paper.

\section{References}

[1] L. Zhang, C. Su, Y. Jin, M. Goh, and Z. Wu, "Cross-network dissemination model of public opinion in coupled networks," Information Sciences, vol. 451-452, pp. 240-252, 2018. 
[2] K. Ma, W. Liu, K. Zhang, Z. Duanmu, Z. Wang, and W. Zuo, "End-to-end blind image quality assessment using deep neural networks," IEEE Transactions on Image Processing, vol. 27, no. 3, pp. 1202-1213, 2018.

[3] Y. Wang, Y. Li, Y. Song, and X. Rong, "The influence of the activation function in a convolution neural network model of facial expression recognition," Applied Sciences, vol. 10, no. 5, p. $1897,2020$.

[4] G. Sun and S. Bin, "A new opinion leaders detecting algorithm in multi-relationship online social networks," Multimedia Tools and Applications, vol. 77, no. 4, pp. 4295-4307, 2018.

[5] W. Zhang, Y. du, T. Yoshida, and Q. Wang, "DRI-RCNN: an approach to deceptive review identification using recurrent convolutional neural network," Information Processing \& Management, vol. 54, no. 4, pp. 576-592, 2018.

[6] W. Chen, Z. Jiang, H. Guo, and X. Ni, "Fall detection based on key points of human-skeleton using openpose," Symmetry, vol. 12 , no. 5 , p. 744, 2020.

[7] C. Li, L. Wang, S. Sun, and C. Xia, "Identification of influential spreaders based on classified neighbors in real-world complex networks," Applied Mathematics and Computation, vol. 320, pp. 512-523, 2018.

[8] B. Liu, Y. Zhang, D. J. He, and Y. Li, "Identification of apple leaf diseases based on deep convolutional neural networks," Symmetry, vol. 10, no. 1, p. 11, 2018.

[9] A. Baker and L. Renno, "Nonpartisans as false negatives: the mismeasurement of party identification in public opinion surveys," The Journal of Politics, vol. 81, no. 3, pp. 906-922, 2019.

[10] F. Rundo, S. Conoci, A. Ortis, and S. Battiato, “An advanced bio-inspired photoplethysmography (PPG) and ECG pattern recognition system for medical assessment," Sensors, vol. 18, no. 2, p. 405, 2018.

[11] M. Kubanek, J. Bobulski, and J. Kulawik, "A method of speech coding for speech recognition using a convolutional neural network," Symmetry, vol. 11, no. 9, p. 1185, 2019.

[12] W. Tao, M. C. Leu, and Z. Yin, "American Sign Language alphabet recognition using convolutional neural networks with multiview augmentation and inference fusion," Engineering Applications of Artificial Intelligence, vol. 76, pp. 202-213, 2018.

[13] Z. Qiu, J. Chen, Y. Zhao, S. Zhu, Y. He, and C. Zhang, "Variety identification of single rice seed using hyperspectral imaging combined with convolutional neural network," Applied Sciences, vol. 8, no. 2, p. 212, 2018.

[14] J. Weismueller, P. Harrigan, S. Wang, and G. N. Soutar, "Influencer endorsements: how advertising disclosure and source credibility affect consumer purchase intention on social media," Australasian Marketing Journal, vol. 28, no. 4, pp. 160-170, 2020.

[15] Z. Hou, F. Cui, Y. Meng, T. Lian, and C. Yu, "Opinion mining from online travel reviews: a comparative analysis of Chinese major OTAs using semantic association analysis," Tourism Management, vol. 74, pp. 276-289, 2019.

[16] Y. Shan, K. J. Chen, and J. S. Lin, "When social media influencers endorse brands: the effects of self-influencer congruence, parasocial identification, and perceived endorser motive," International Journal of Advertising, vol. 39 , no. 5, pp. 590$610,2020$.

[17] J. T. Jost, P. Barberá, R. Bonneau et al., "How social media facilitates political protest: information, motivation, and social networks," Political Psychology, vol. 39, pp. 85-118, 2018.
[18] M. D. R. Evans and J. Kelley, "Strong welfare states do not intensify public support for income redistribution, but even reduce it among the prosperous: a multilevel analysis of public opinion in 30 countries," Societies, vol. 8, no. 4, p. 105, 2018.

[19] I. Chaturvedi, R. Satapathy, S. Cavallari, and E. Cambria, "Fuzzy commonsense reasoning for multimodal sentiment analysis," Pattern Recognition Letters, vol. 125, pp. 264-270, 2019.

[20] S. Lewandowsky, T. D. Pilditch, J. K. Madsen, N. Oreskes, and J. S. Risbey, "Influence and seepage: an evidence-resistant minority can affect public opinion and scientific belief formation," Cognition, vol. 188, pp. 124-139, 2019.

[21] L. V. Casaló, C. Flavián, and S. Ibáñez-Sánchez, "Influencers on Instagram: antecedents and consequences of opinion leadership," Journal of Business Research, vol. 117, pp. 510-519, 2020 . 